\title{
Integrated analysis reveals potential long non-coding RNA biomarkers and their potential biological functions for disease free survival in gastric cancer patients
}

\author{
Canchang Cheng ${ }^{1 \dagger}$, Qicai Wang ${ }^{2 \dagger}$, Minggu Zhu ${ }^{1}$, Kelong Liu ${ }^{2}$ and Zhiqiao Zhang ${ }^{1 *}$ (D)
}

\begin{abstract}
Background: Increasing evidences supported the association between long non-coding RNA (IncRNA) and disease free survival in gastric cancer (GC) patients. The purpose of the current study was to construct and verify a noninvasive preoperative predictive tool for disease free survival in GC patients.

Methods: There were 265 and 300 GC patients in model dataset and validation dataset respectively. The associations between the IncRNA biomarkers and disease free survival were evaluated by univariate and multivariate Cox regression.

Results: Thirteen IncRNA biomarkers (GAS5-AS1, AL109615.3, KDM7A-DT, AP000866.2, KCNJ2-AS1, LINC00656, LINC01777, AC046185.3, TTTY14, LINC01526, LINC02523, LINC00592, and C5orf66) were identified as prognostic biomarkers with disease free survival. These thirteen IncRNA biomarkers were combined to construct a prognostic signature for disease free survival. The C-indexes of the current predictive signature in model cohort were 0.849 (95\% Cl 0.803-0.895), 0.859 (95\% Cl 0.813-0.905) and 0.888 (95\% Cl 0.842-0.934) for 1-year, 3-year and 5-year disease free survival respectively. Based on thirteen-IncRNA prognostic signature, patients in model cohort could be stratified into high risk group and low risk group with significant different disease free survival rate (hazard ratio $[H R]=7.355,95 \%$ confidence interval [CI] 4.378-12.356). Good reproducibility of thirteen-IncRNA prognostic signature was confirmed in an external validation cohort (GSE62254) with HR 3.919 and 95\% Cl 2.817-5.453. Further analysis demonstrated that the prognostic significance of thirteen-IncRNA prognostic signature was independent of other clinical characteristics.

Conclusions: In conclusion, a simple noninvasive prognostic signature was established for preoperative prediction of disease free survival in GC patients. This prognostic signature might predict the individual mortality risk of disease free survival without pathological information and facilitate individual treatment decision-making.
\end{abstract}

Keywords: Long non-coding RNA, IncRNA, Gastric cancer, Disease free survival, Prognostic signature

\footnotetext{
*Correspondence: sdgrxjbk@163.com

${ }^{\dagger}$ Canchang Cheng and Qicai Wang made the same contribution to

the current research and therefore were the parallel first authors of the current manuscript

${ }^{1}$ Department of Internal Medicine, The Affiliated Chencun Hospital of Shunde Hospital, Southern Medical University, Shunde District,

Guangdong, China

Full list of author information is available at the end of the article
}

(c) The Author(s) 2019. This article is distributed under the terms of the Creative Commons Attribution 4.0 International License (http://creativecommons.org/licenses/by/4.0/), which permits unrestricted use, distribution, and reproduction in any medium, provided you give appropriate credit to the original author(s) and the source, provide a link to the Creative Commons license, and indicate if changes were made. The Creative Commons Public Domain Dedication waiver (http://creativecommons.org/ publicdomain/zero/1.0/) applies to the data made available in this article, unless otherwise stated. 


\section{Introduction}

As a serious challenge to public health, gastric cancer (GC) is the fifth most common cancer and the third leading cause for cancer associated mortality in the world [1]. It was estimated that there were approximately 1,033,701 GC patients occurred and 782,685 GC patients died in 2018 [1]. Despite the improvements of diagnosis and treatments, the prognosis of GC patients remains undesirable $[2,3]$. The TNM stage system of the American Joint Committee on Cancer (AJCC) was insufficient for prognostic prediction of GC patients [4, 5]. Increasing evidences demonstrated that the molecular biomarkers were helpful for improvement of prognostic prediction and early identification of GC patients with high mortality risk [6-8]. Thus, it is necessary to develop a valuable preoperative prognostic signature to identify GC patients with high mortality risk and improve the clinical treatment decision.

Long non-coding RNAs (lncRNAs) are RNAs that length range from 200 nucleotides to multiple kilobases but lack of protein-coding function [9]. Emerging evidences have revealed that lncRNAs could provide valuable prognostic information for GC patients [10-12]. Several studies have developed lncRNA-based prognostic signatures for disease free survival (DFS) in GC patients [13-15]. However, these lncRNA-based prognostic signatures had several limitations for preoperative prediction of disease free survival: Firstly, the calculation formulas of these lncRNA-based prognostic signatures were too complex for clinical application. Secondly, the prognostic significances of absolute scores of these previous prognostic signatures were difficult to understand and interpret for users without medical knowledge. Thirdly, these three prognostic signatures lacked external validation. Fourthly, Tian et al. constructed a lncRNA-based prognostic signature for 3-year DFS by combination of data of gene expression and pathological parameters. However, for patients with advanced GC cancer or who were unwilling to undergo surgery, the pathological parameters were unobtainable for calculation of prognostic signature and thus seriously limited the clinical application of this prognostic signature. Thus, it is necessary to develop and validate a simple noninvasive signature for preoperative prediction of prognosis in GC patients.

Nomogram was a method of displaying calculation results by percentage scale diagram and was used for prognostic prediction in different cancer patients $[8,16]$. Nomogram could easily attain the predictive percentage of study outcome without complex calculation. Therefore, to construct a simple prognostic signature for disease free survival in GC patients, the current study developed and validated a prognostic signature by using nomogram method. We carried out the current study and reported the results in accordance with the guidelines of Transparent Reporting of a multivariable prediction model for individual prognosis or diagnosis (TRIPOD) [17].

\section{Materials and methods Protocol approval}

The study datasets in the current study were obtained from The Cancer Genome Atlas (TCGA) database (https://cancergenome.nih.gov/) and the Gene Expression Omnibus (GEO) database (https://www.ncbi.nlm. nih.gov/gds/). The current study downloaded and analyzed the study datasets according to the data policies of TCGA database and GEO database. The study datasets obtained from TCGA database and GEO database were fully anonymous and therefore the ethics approval was not required.

\section{The model dataset}

The model dataset was downloaded from TCGA database (https://tcga-data.nci.nih.gov/docs/publications/ tcga/). The gene expression values were generated by using the Illumina HiSeq 2000 RNA Sequencing platform. In the current study, the selected lncRNA IDs were defined according to GENCODE Version 29 (https ://www.gencodegenes.org/human/). Finally, there were 14,449 lncRNAs obtained from 375 tumor specimens and 32 normal specimens in model dataset. The original gene expression counts were converted to 0 for low expression and 1 for high expression according to the median values of original gene expression values. The clinical information of $443 \mathrm{GC}$ patients in model dataset were downloaded from cBioPortal database (http:// www.cbioportal.org/datasets). The GC patients without disease free survival information were excluded from the current study $(n=120)$. In order to avoid or reduce the interferences of confounding factors, there were $10 \mathrm{GC}$ patients excluded from the current study because disease free survival time less than 1 month $(\mathrm{n}=10)$. After interaction between gene dataset and clinical dataset, there were $265 \mathrm{GC}$ patients with gene expression information and survival information included in the model cohort (Fig. 1). The maximum DFS time was 122.21 months and the minimum DFS time was 1.02 month in model cohort. The study time was from January 13, 2002 to November 24,2014 . The missing data were coded as "NA" in the model dataset. The mean \pm standard deviation (SD) age of GC patients in the model cohort was $64.4 \pm 10.6$ years. Ninety-eight (37.0\%) patients out of 265 GC patients died within the follow-up period (mean \pm SD: $618 \pm 564$ days).

\section{The validation dataset}

We explored and identified potential study datasets in GEO database according to the following criteria: (1) 
The original gene expression information of model cohort contained 15240 IncRNA expression information from 375 cancer samples and 32 adjacent normal tissues $(n=407)$.

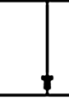

Of 443 patients in model dataset downloaded from cBioPortal database, 120 patients with adequate disease free survival information and 10 Patients with disease free survival time less than 1 month were excluded from the current study. Finally, there were 313 patients with enough clinical survival information in model group ( $n=313)$.
The gene expression information of validation cohort contained 54675 probe IDs detected on Affymetrix Human Genome U133 Plus 2.0 chip platform ( $n=300)$.

The clinical information of 300
patients in validation dataset were
downloaded from GEO database.
There were 300 patients with
enough clinical survival information
in validation group $(n=300)$.
enough clinical survival information

\section{t}

After gene symbol intersection between model cohort and validation cohort, there were 265 patients in model cohort and 300 patientsand in validation cohort with 1978 same IncRNA.

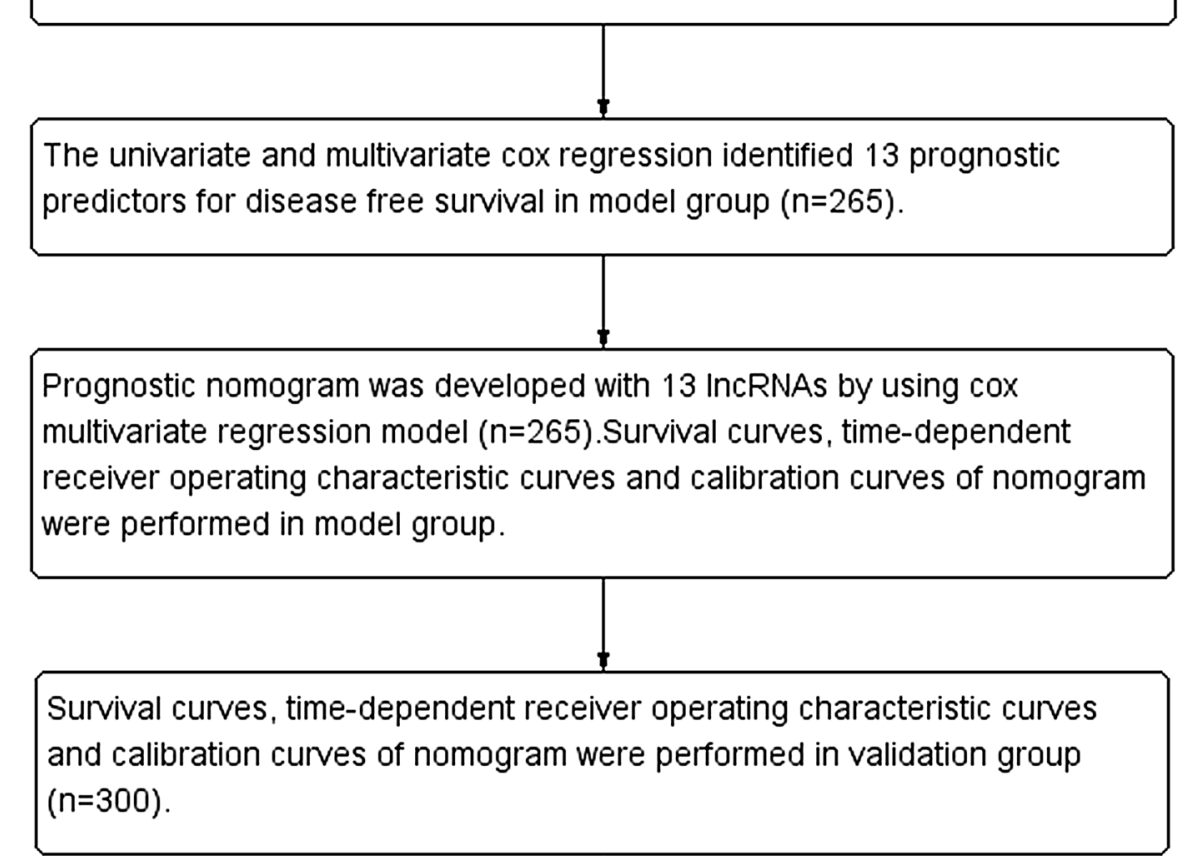

Fig. 1 The flowchart in the current study. TCGA, The Cancer Genome Atlas

gene expression values detected by using the Affymetrix Human Genome U133 Plus 2.0 array; (2) DFS time and DFS status were provided in the corresponding studies; (3) patient number more than 100. Finally, we identified
GSE62254 as an independent external validation dataset [18]. The gene expression information and corresponding clinical information were downloaded from the GEO database (http://www.ncbi.nlm.nih.gov/geo/). There were 
300 GC patients with gene expression information and clinical information from GSE62254 (https://www.ncbi. nlm.nih.gov/geo/query/acc.cgi?acc=GSE62254). The gene expression information was detected on Affymetrix Human Genome U133 Plus 2.0 chip platform. The patient selection flow chart was described in Fig. 1. The Affymetrix HG-U133 Plus 2.0 probe set IDs were mapped to the Ensembl gene IDs by using the platform background file of GPL570 (https://www.ncbi.nlm.nih.gov/geo/query /acc.cgi?acc $=$ GPL570).

Based on the lncRNA IDs defined in GENCODE Version 29 (https://www.gencodegenes.org/human/), 1978 lncRNAs with corresponding gene symbols from $300 \mathrm{GC}$ patients were extracted and analyzed for further survival study.

\section{Development and validation of the prognostic nomogram}

The prognostic nomogram and the corresponding calibration plots were generated by using "rms" package of $\mathrm{R}$ software. Calibration plots were performed to evaluate the predictive performance of the prognostic nomogram. The predicted survival and observed survival were plotted on the $\mathrm{x}$-axis and $\mathrm{y}$-axis respectively. The 45-degree line represented the best predictive curve. Time-dependent ROC curves were conducted to assess the predictive performance of the prognostic nomogram by using the "pROC" package.

\section{The decision curve analysis}

The decision curve analysis (DCA) was performed to evaluate the clinical utility of the prognostic nomogram for disease free survival. The DCA is a method for evaluation and comparison of the predictive value between different prediction models [19-21]. The $\mathrm{x}$-axis of DCA represented the percentage of threshold probability, and the $y$-axis represented the net benefit of the predictive model. The net benefit was calculated according to the following formula: Net benefit $=$ (True positives $/ \mathrm{n})-($ False positives $/ \mathrm{n}){ }^{*}\left(\mathrm{p}_{\mathrm{t}} /\left(1-\mathrm{p}_{\mathrm{t}}\right)\right.$.

\section{Functional enrichment analyses}

To explore the potential biological functions of lncRNAs included in the prognosis signature, the co-expressed mRNA were obtained through the model dataset according to the thresholds of $P$ value $<0.05$ and |spearman correlation coefficient $\mid>0.5$. Functional enrichment analyses of these co-expressed mRNAs were performed by using the Database for Annotation, Visualization and Integrated Discovery (DAVID, https://david.ncifcrf.gov/) [22].

\section{Statistical analysis}

Continuous variables were displayed as mean \pm standard deviation (SD). Continuous variables were compared by $t$ test or Mann-Whitney U test as appropriate. Categorical variables were compared by using Chi squared test or Fisher's exact test as appropriate. To explore the potential associations between lncRNAs and DFS, univariate Cox regression analyses and multivariate Cox regression analyses were performed to identify the prognostic biomarkers for development of prognosis signature. The GC patients were divided into two subgroups according to the scores generated by the prognosis signature. KaplanMeier analysis was used to compare the difference of DFS between high risk group and low risk group. The predictive performance and clinical utility of prognostic signatures were evaluated by using the Harrell's concordance index and time-dependent receiver operating characteristic (ROC) curve. The statistical analyses in the present study were conducted by SPSS Statistics 19.0 (SPSS Inc., an IBM Company) and R software (version 3.4.5). The R packages, including "pROC", "plyr", "rms", "survival", "timeROC " and "glmnet ", were used as needed in the current study. $P$ value $<0.05$ was defined as statistically significance in the current study.

\section{Results}

\section{Study cohort}

The flow chart of patient selection in the current study was showed in Fig. 1. There were 265 GC patients in the model group (Additional file 1) and 300 GC patients in the validation group (Additional file 2). There were 98 (37.0\%) patients out of 265 patients died within the follow-up period in the model group, whereas there were $161(53.7 \%)$ patients out of 300 patients died within the follow-up period in the validation group. The basic clinical characteristics of GC patients in the model group and validation group were presented in Table 1.

\section{Development of prognostic signature}

The univariate Cox proportional regression analyses were carried out to explore the potential lncRNA predictors for disease free survival in GC patients. There were 249 lncRNAs that were significantly related with DFS in the model group. According to the multivariate Cox regression analyses, there were 13 lncRNAs identified as independent biomarkers for disease free survival in GC patients. The relevant estimated regression coefficients of these 13 prognostic lncRNAs were showed in Table 2. Therefore, a prognostic signature was conducted according to the following formula: Prognostic signature score $=(-0.712 *$ GAS5$\mathrm{AS} 1)+\left(-0.725^{*} \mathrm{AL} 109615.3\right)+(-0.830 * \mathrm{KDM} 7 \mathrm{~A}-$ 
Table 1 The clinical features of gastric cancer patients in the model group and validation group

\begin{tabular}{|c|c|c|c|}
\hline & Model group $(n=265)$ & Validation group $(n=300)$ & $P$ value \\
\hline Death [n (\%)] & $98(37.0)$ & $161(53.7)$ & $<0.001$ \\
\hline Survival time (mean $\pm S D$, month) & $20.6 \pm 18.8$ & $33.7 \pm 29.8$ & 0.008 \\
\hline Age (mean $\pm S D$, year) & $64.4 \pm 10.6$ & $61.9 \pm 11.4$ & $<0.001$ \\
\hline Male $[(n) \%]$ & $175(66.0)$ & $199(66.3)$ & 0.941 \\
\hline AJCC Stage (IV/III/II/I/NA) & 23/103/91/41/7 & $77 / 95 / 98 / 30 / 0$ & $<0.001$ \\
\hline AJCC PT (T4/T3/T2/T1/NA) & $66 / 121 / 62 / 16 / 0$ & 21/91/188/0/0 & $<0.001$ \\
\hline AJCC PN (N4/N3/N2/N1/NO/NA) & $4 / 49 / 55 / 67 / 88 / 2$ & 0/51/80/131/38/0 & 0.011 \\
\hline AJCC PM (M2/M1/M0/NA) & $9 / 13 / 243 / 0$ & 0/27/273/0 & 0.869 \\
\hline Targeted molecular therapy (yes/no/NA) & 78/79/108 & NA & \\
\hline Radiation treatment adjuvant (yes/no/NA) & $0 / 155 / 110$ & NA & \\
\hline History other malignancy (yes/no/NA) & $6 / 259 / 0$ & NA & \\
\hline Barretts esophagus (yes/no/NA) & $12 / 151 / 102$ & NA & \\
\hline H pylori infection (yes/no/NA) & $15 / 117 / 133$ & NA & \\
\hline
\end{tabular}

Continuous variables were compared by t-test or Mann-Whitney $\mathrm{U}$ test as appropriate; categorical variables were compared by Chi squared test or Fisher's exact test as appropriate

NA, missing data; SD: standard deviation; AJCC: American Joint Committee on Cancer

Table 2 The model information of prognostic IncRNA predictors in univariate and multivariable Cox regression analyses

\begin{tabular}{|c|c|c|c|c|c|c|c|}
\hline \multirow[t]{2}{*}{ Variables } & \multicolumn{3}{|c|}{ Univariate analysis } & \multicolumn{4}{|c|}{ Multivariate analysis } \\
\hline & $\mathrm{HR}$ & $95 \% \mathrm{Cl}$ & $P$-value & Coefficient & HR & $95 \% \mathrm{Cl}$ & $P$-value \\
\hline GAS5-AS1 (high/low) & 0.580 & $0.387-0.870$ & 0.008 & -0.712 & 0.491 & $0.319-0.755$ & 0.001 \\
\hline AL109615.3 (high/low) & 0.632 & $0.422-0.948$ & 0.027 & -0.725 & 0.485 & $0.315-0.744$ & 0.001 \\
\hline KDM7A-DT (high/low) & 0.628 & $0.420-0.939$ & 0.024 & -0.830 & 0.436 & $0.275-0.690$ & $<0.001$ \\
\hline AP000866.2 (high/low) & 0.633 & $0.423-0.947$ & 0.026 & -0.837 & 0.433 & $0.276-0.677$ & $<0.001$ \\
\hline KCNJ2-AS1 (high/low) & 0.642 & $0.429-0.959$ & 0.031 & -0.984 & 0.374 & $0.237-0.588$ & $<0.001$ \\
\hline LINC00656 (high/low) & 0.651 & $0.436-0.973$ & 0.036 & -1.150 & 0.317 & $0.199-0.503$ & $<0.001$ \\
\hline LINC01777 (high/low) & 1.907 & $1.260-2.886$ & 0.002 & 0.764 & 2.147 & $1.354-3.403$ & 0.001 \\
\hline AC046185.3 (high/low) & 1.566 & $1.049-2.339$ & 0.028 & 0.769 & 2.157 & $1.373-3.388$ & 0.001 \\
\hline TTTY14 (high/low) & 1.786 & $1.191-2.678$ & 0.005 & 0.775 & 2.170 & $1.412-3.333$ & $<0.001$ \\
\hline LINC01526 (high/low) & 1.669 & $1.115-2.500$ & 0.013 & 0.783 & 2.188 & $1.397-3.425$ & 0.001 \\
\hline LINC02523 (high/low) & 1.890 & $1.252-2.852$ & 0.002 & 0.803 & 2.232 & $1.373-3.625$ & 0.001 \\
\hline LINC00592 (high/low) & 1.594 & $1.066-2.382$ & 0.023 & 0.941 & 2.563 & $1.658-3.963$ & $<0.001$ \\
\hline C5orf66 (high/low) & 1.505 & $1.005-2.254$ & 0.047 & 1.162 & 3.195 & $2.001-5.105$ & $<0.001$ \\
\hline
\end{tabular}

The medians of IncRNA expression values were used as cut-off values to stratify IncRNA expression values into high expression group (as value 1) and low expression group (as value 0 )

$\mathrm{HR}$, hazard ratio; $\mathrm{Cl}$, confidence interval

$\mathrm{DT})+(-0.837 * \mathrm{AP} 000866.2)+(-0.984 * \mathrm{KCNJ} 2-$ AS1 $)+(-1.150 *$ LINC00656) $+(0.764 *$ LINC01777 $)+(0.7$ $69 * \mathrm{AC} 046185.3)+(0.775 * \mathrm{TTTY} 14)+(0.783 * \mathrm{LINC01526})$ $+(0.803 *$ LINC02523) $+(0.941 *$ LINC00592) $+(1.162 *$ C5o rf66). According to the multivariate Cox regression analyses, a prognostic nomogram for prediction of disease free survival in gastric cancer patients was presented in Fig. 2.

This nomogram could be used to predict the individual mortality risk. The value of each lncRNA was calculated according to the corresponding scale. The total score was obtained by adding the values of these thirteen lncRNA. The total score was projected to the probability of DFS in 1 year, 3 year, and 5 year respectively.

Distribution characteristics of prognostic signature score For displaying the distribution characteristics of prognostic signature score, the violin plot (Fig. 3a), density plot (Fig. 3b), scatter plot (Fig. 4a), the interaction distribution scatter plot among DFS time, DFS status, and predictive value (Fig. 4b) were presented in Figs. 3 and 4. 


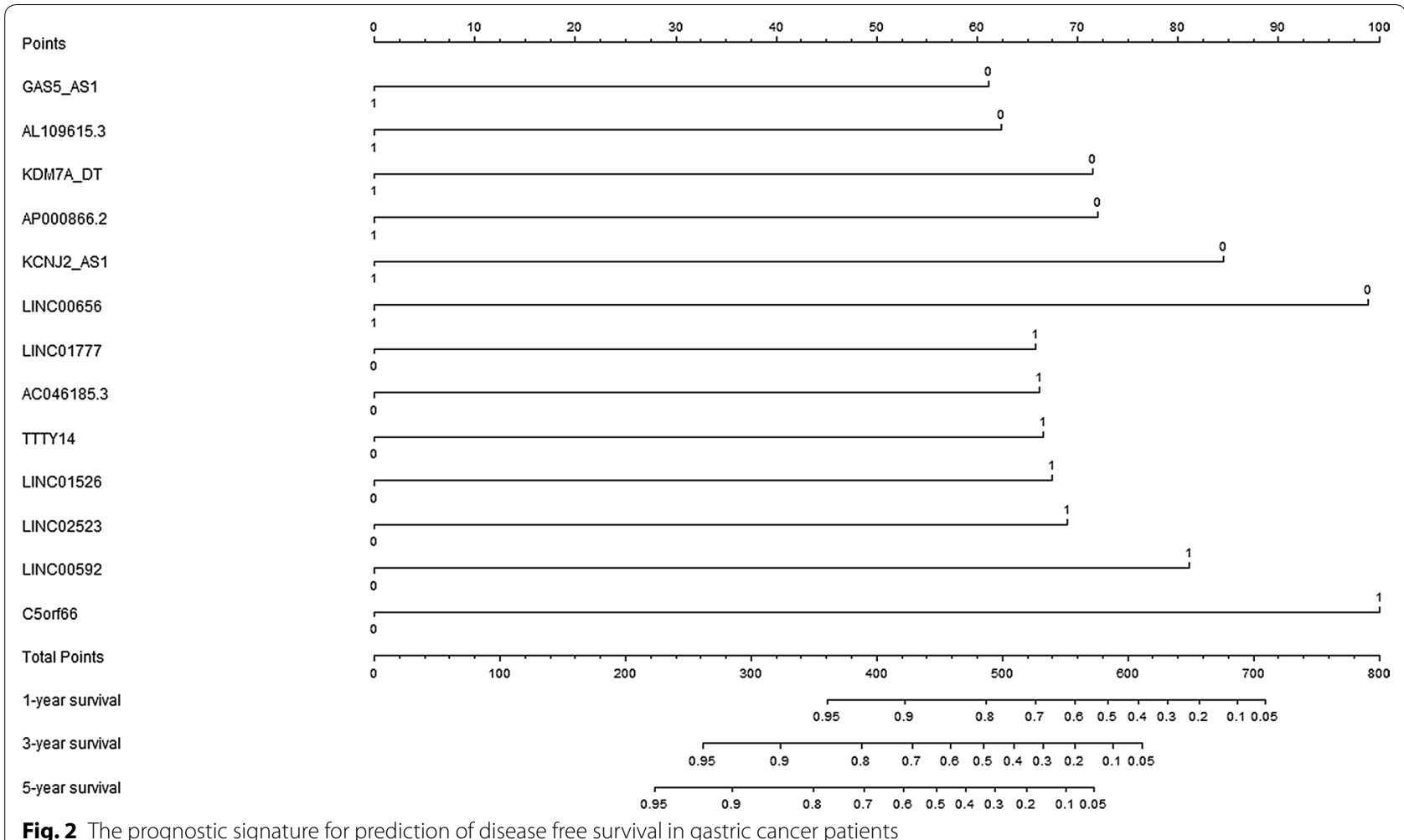

Fig. 2 The prognostic signature for prediction of disease free survival in gastric cancer patients
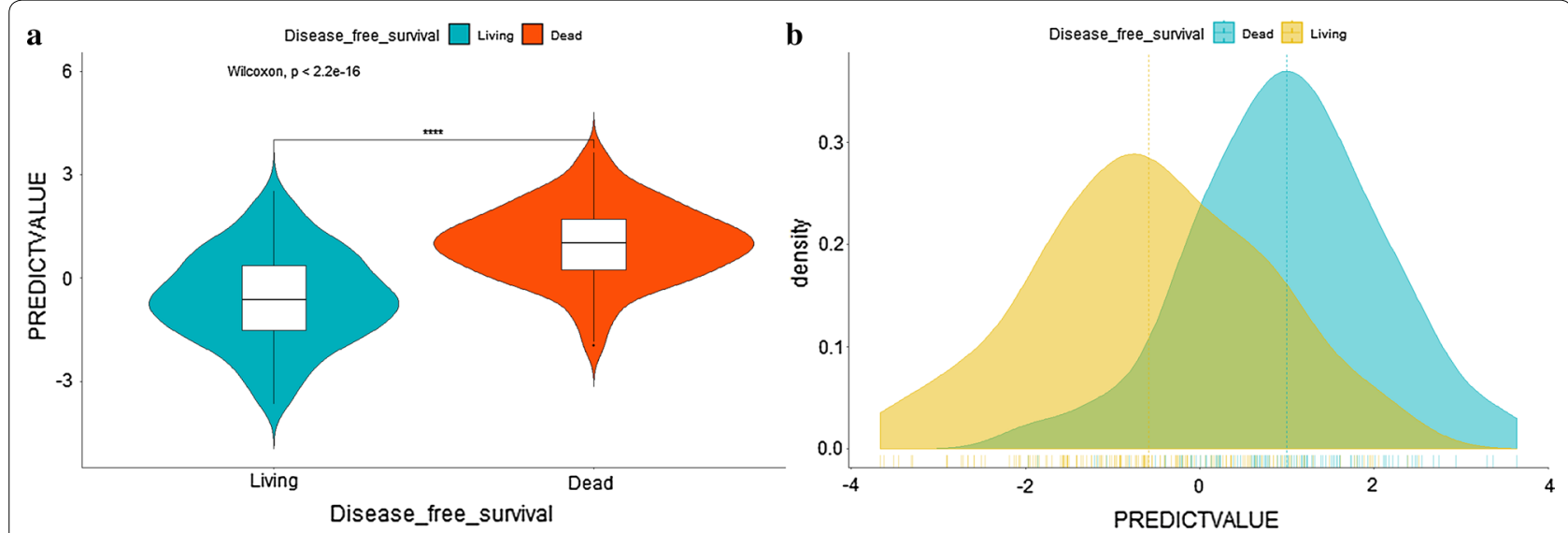

Fig. 3 Distribution characteristics of prognostic signature score in the model group: the violin plot of prognostic signature score (a); the density plot of prognostic signature score (b)

\section{Clinical utility of prognostic signature}

According to the median value of prognostic signature score, GC patients in model cohort $(\mathrm{n}=265)$ were stratified into high risk group $(\mathrm{n}=133)$ and low risk group $(\mathrm{n}=132)$. The disease free survival rate (Fig. 5a) in high risk group was significantly poorer than that in low risk group $(P<0.001)$. The cumulative proportion surviving at 1 -year, 3 -year, and 5 -year were $94.3 \%, 81.7 \%$, and $77.1 \%$ in low risk group, whereas it were $59.2 \%, 27.8 \%$ and $11.3 \%$ in high risk group respectively (all $P<0.001$ ). The Harrell's concordance indexes (C-indexes) of prognostic signature for disease free survival in the model group were 0.849 (95\% CI 0.803-0.895), 0.859 (95\% CI 0.813-0.905) and 0.888 (95\% CI $0.842-0.934)$ for 1-year disease free survival, 3-year disease free survival and 5-year disease free survival respectively (Fig. 5b). The calibration curves 

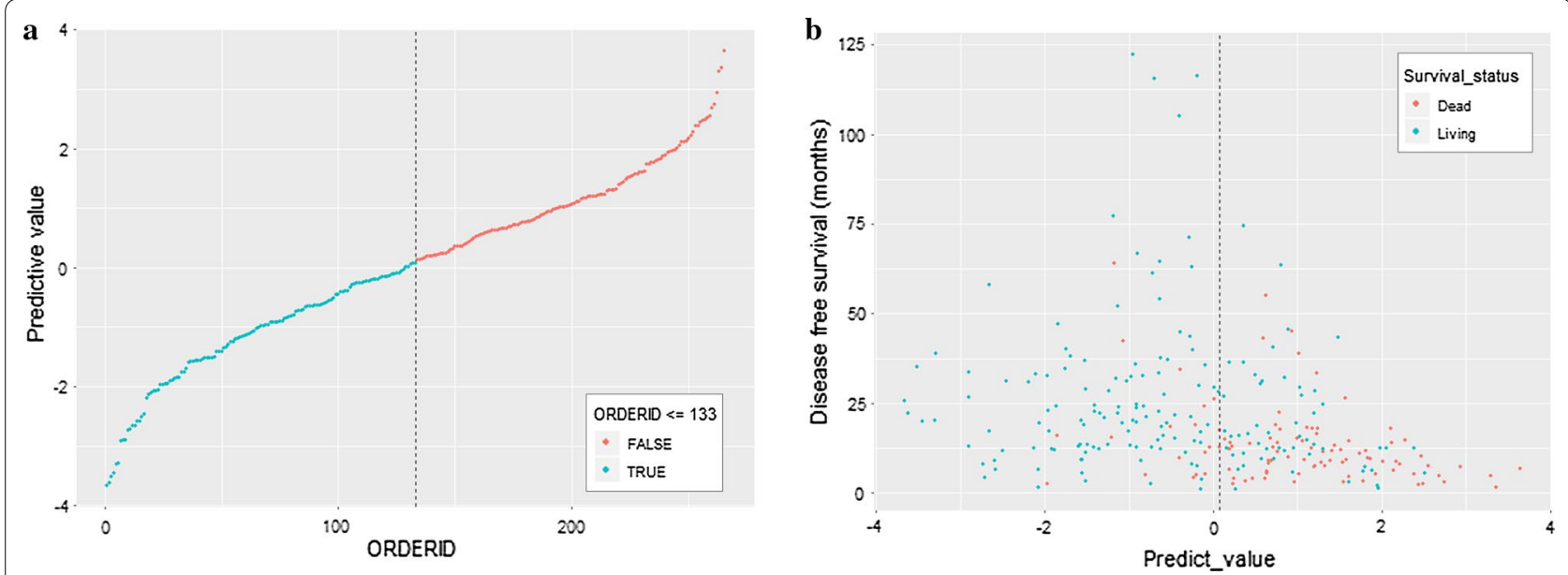

Fig. 4 The distribution of prognostic signature score in the model group (a); the disease free survival status and disease free survival time in the model group (b)

for 1-year, 3-year, and 5-year disease free survival were presented in Fig. 6.

\section{External validation of prognostic signature}

We validated the clinical utility of prognostic signature by using GSE62254 dataset. The prognostic signature scores for GC patients in validation dataset were calculated according to the previous prognosis signature formula in model dataset. Then the GC patients in validation set $(n=300)$ were divided into low risk group $(\mathrm{n}=150)$ and high risk group $(\mathrm{n}=150)$. The cumulative proportion surviving at 1-year, 3-year, and 5-year were $89.3 \%, 70.1 \%$, and $62.3 \%$ in low risk group, whereas it were $42.4 \%, 24.1 \%$ and $21.4 \%$ in high risk group respectively (all $P<0.001$ ). Kaplan-Meier analysis (Fig. 7a) indicated that there was significant difference in term of DFS between low risk group and high risk group in validation set $(P<0.001)$. The $C$-index of prognostic signature for disease free survival in the validation group were 0.870 (95\% CI 0.834-0.906), 0.822 (95\% CI 0.786-0.858) and 0.816 (95\% CI 0.780-0.894) for 1-year, 3-year, and 5 -year disease free survival respectively (Fig. 7b). The calibration curves for 1-year, 3-year, and 5-year disease free survival were presented in Fig. 8 for validation cohort.

\section{Independence assessment for prognostic significance of prognostic signature}

We carried out multivariate Cox regression analyses to explore whether prognostic signature was independent to other clinical parameters for DFS in GC patients. The pathological diagnosis was performed according to the suggestions of American Joint Committee on Cancer (AJCC). Table 3 indicated that prognostic signature was an independent risk factor for DFS after adjustment for confounding effects of gender, age, and pathological stage in the model group. In the validation group, multivariate Cox regression analyses demonstrated that prognostic signature, age, and AJCC stage were independent risk factors for DFS.

\section{Functional enrichment analyses}

According to a threshold of $P$ value $<0.05$ and | spearman correlation coefficient $\mid>0.5$, there were $1280 \mathrm{mRNA}$ genes that significantly co-expressed with the prognostic lncRNAs in the prognostic signature. Functional enrichment analyses were carried out through the Database for Annotation, Visualization, and Integrated Discovery (DAVID) Bioinformatics Resources (https://david .ncifcrf.gov/). The gene ontology (GO) biological process enrichment analyses and the Kyoto Encyclopedia of Genes and Genomes (KEGG) signaling pathways were presented in Fig. 9. Functional enrichment analyses of the 1280 co-expressed mRNA genes demonstrated that the co-expressed mRNA genes were mainly enriched in regulation of transcription, RNA splicing, protein ubiquitination, cellular response to DNA damage stimulus, cilium assembly, cilium morphogenesis, centrosome organization, G2/M transition of mitotic cell cycle, regulation of transcription from RNA polymerase II promoter.

\section{The decision curve analysis (DCA)}

As shown in Fig. 10, the prognostic signature (red line) had the higher net benefit than the pathological stage (green line). The decision curve analyses indicated that prognostic signature could gain more benefit than either the dead-all-patients scheme or the dead-nonepatients scheme for prediction of 1-year DFS (Fig. 10a), 

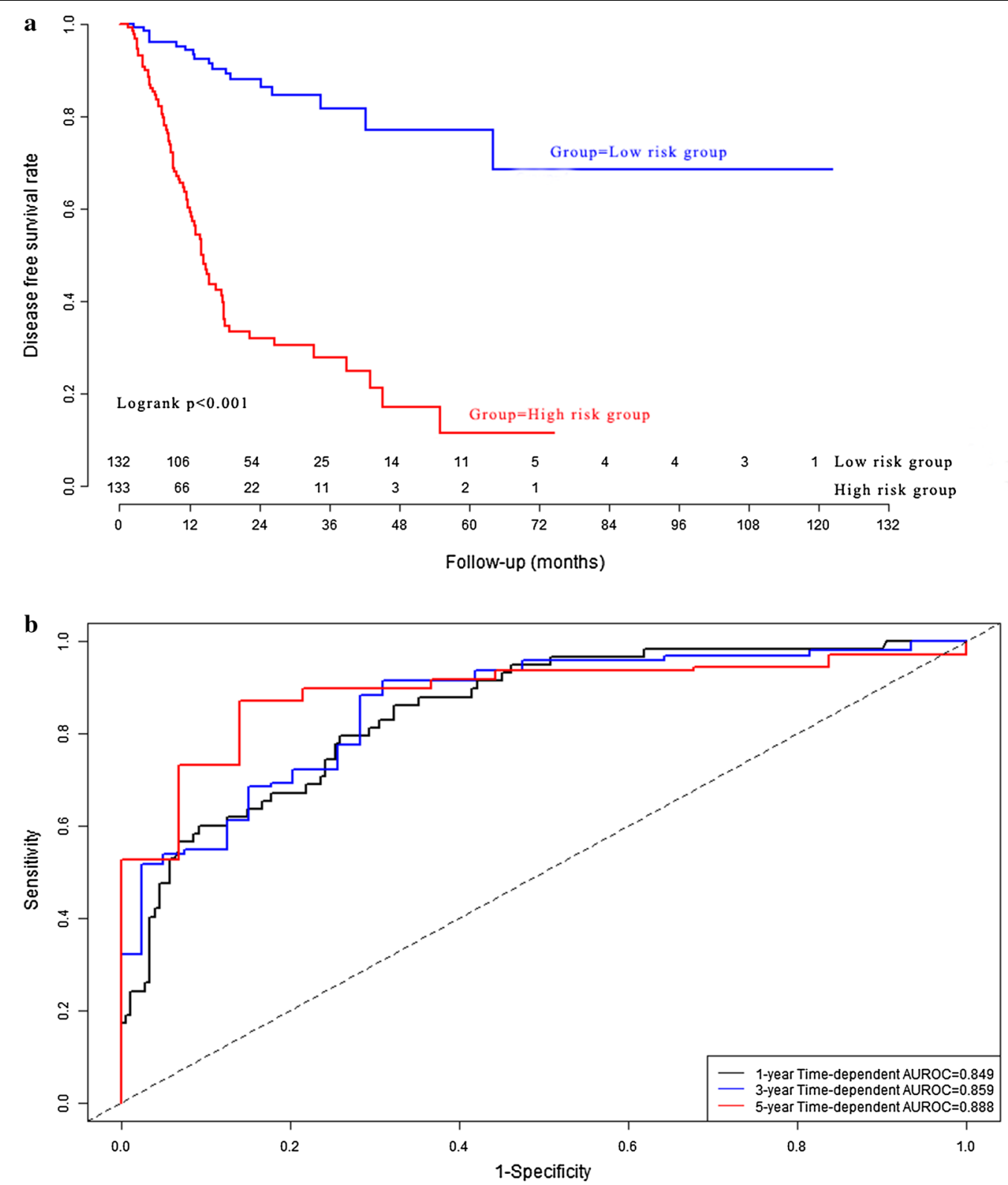

Fig. 5 Performance of prognostic signature in the model group: the survival curves of gastric cancer patients (a); time-dependent receiver operating characteristic curves (b)

3-year DFS (Fig. 10b), and 5-year DFS (Fig. 10c). Clinical impact curve (Fig. 10d) depicted the prediction of risk stratification of 1000 patients by using resample bootstrap method. "Number high risk" indicated the number of patients classified as positive (high risk) by prognostic signature according to various threshold probabilities. "Number high risk with event" was the true positive patient number according to various threshold probabilities.

\section{Ten-group risk stratification chart}

To explore the predictive performance of prognostic signature for DFS, a 10-group risk stratification chart was presented in Fig. 11. For model cohort, the discriminative ability of prognostic signature for 1 year, 2 year, and 3 year DFS were showed in Fig. 11a-c. For validation cohort, the discriminative ability of prognostic signature for 1 year, 2 year, and 3 year DFS were showed in Fig. 11d-f. Figure 11 demonstrated that patients in lower 

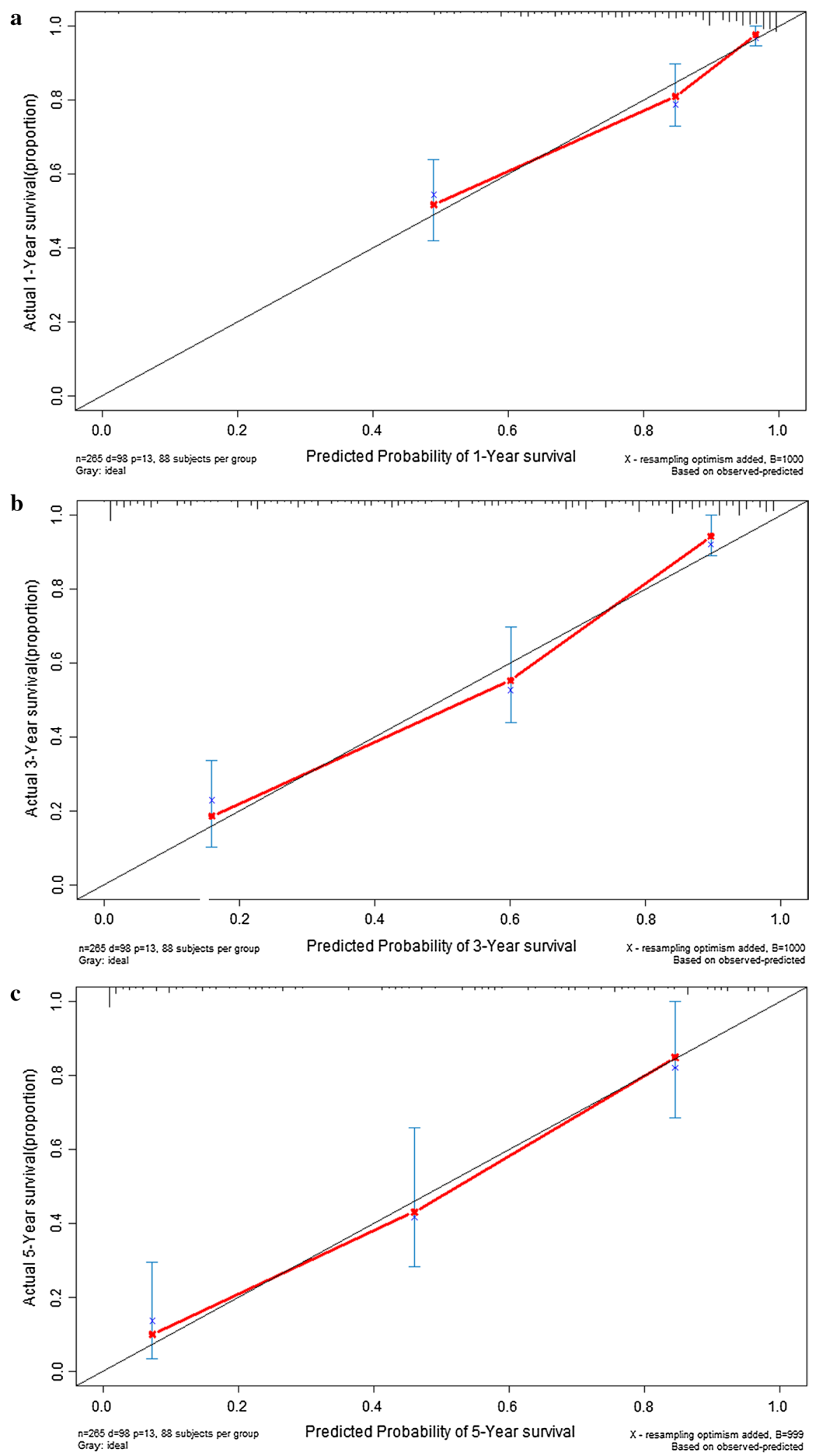

Fig. 6 Calibration curve of prognostic signature in the model group: calibration curve for 1-year disease free survival (a); calibration curve for 3-year disease free survival (b); calibration curve for 5 -year disease free survival (c) 

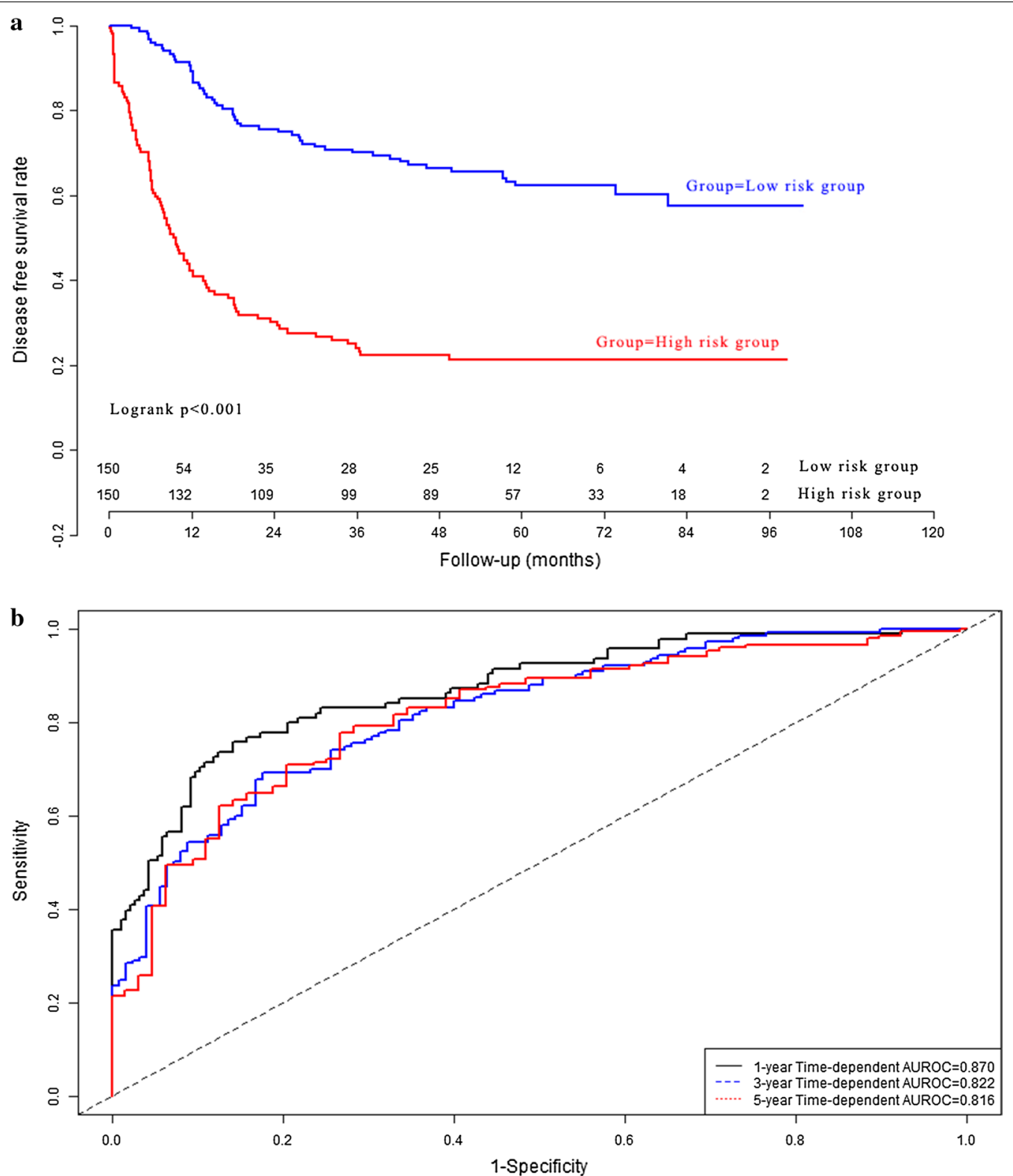

Fig. 7 Performance of prognostic signature in the validation group: the survival curves of gastric cancer patients (a); time-dependent receiver operating characteristic curves (b)

risk groups had higher survival probability and patients in higher risk groups had lower survival probability.

\section{Discussion}

Accurate and reliable prognostic prediction is of critical importance for individualized treatment decisionmaking of GC patients. The current study developed and validated a thirteen-lncRNA prognosis signature for prognostic prediction of GC patients. The thirteenlncRNA prognostic signature was proved to be helpful for individual mortality risk prediction and risk stratification of GC patients in an independent external dataset.

In the current study, the thirteen-IncRNA prognosis signature scores were calculated for prediction of DFS in GC patients in both model set and validation set. Poorer DFS were significantly related with high prognosis signature scores in both model set and validation set, 

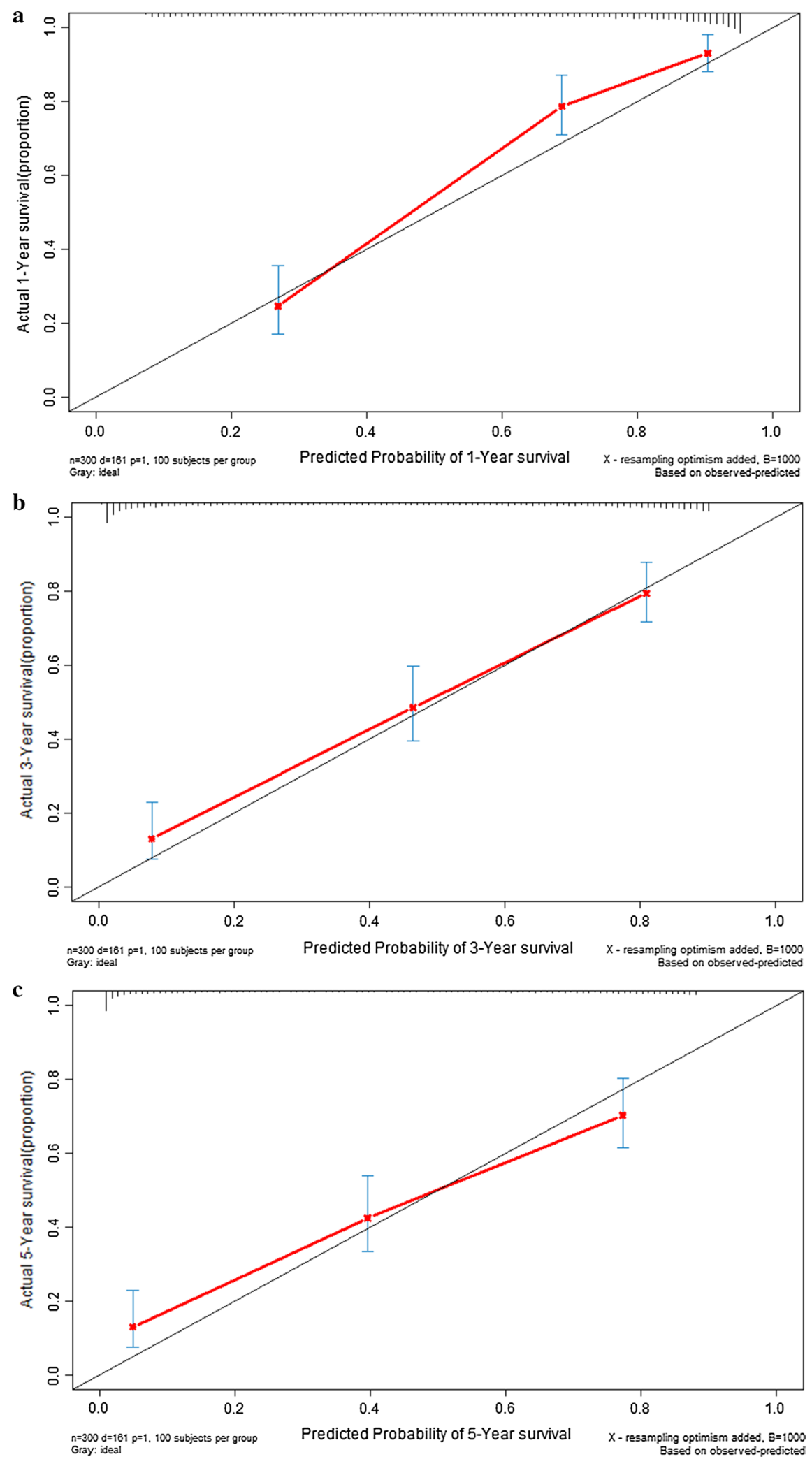

Fig. 8 Calibration curve of prognostic signature in the validation group: calibration curve for 1-year disease free survival (a); calibration curve for 3-year disease free survival (b); calibration curve for 5-year disease free survival (c) 
Table 3 Univariate and multivariable Cox regression analyses for independence assessment of prognostic signature

\begin{tabular}{|c|c|c|c|c|c|c|c|c|}
\hline & \multirow[t]{2}{*}{$\mathbf{n}$} & \multicolumn{3}{|c|}{ Univariate analysis } & \multicolumn{4}{|c|}{ Multivariate analysis } \\
\hline & & HR & $95 \% \mathrm{Cl}$ & $P$-value & Coefficient & HR & $95 \% \mathrm{Cl}$ & $P$-value \\
\hline \multicolumn{9}{|l|}{ Model group $(n=265)$} \\
\hline Age (high/low) & 265 & 0.946 & $0.636-1.408$ & 0.784 & 0.522 & 1.686 & $1.103-2.577$ & 0.016 \\
\hline Gender (male/female) & 265 & 1.872 & $1.173-2.988$ & 0.009 & 0.463 & 1.589 & $0.992-2.547$ & 0.054 \\
\hline AJCC PT (T4,T3/T2, T1) & 265 & 1.385 & $0.876-2.191$ & 0.164 & -0.084 & 0.919 & $0.546-1.547$ & 0.752 \\
\hline AJCC PN (yes/N0) & 265 & 1.639 & $1.034-2.599$ & 0.036 & 0.121 & 1.128 & $0.596-2.135$ & 0.711 \\
\hline AJCC PM (yes/M0) & 265 & 1.056 & $0.512-2.178$ & 0.883 & 0.208 & 1.231 & $0.574-2.637$ & 0.594 \\
\hline AJCC stage (IV, III/II, I) & 265 & 1.607 & $1.072-2.409$ & 0.022 & 0.234 & 1.264 & $0.690-2.314$ & 0.448 \\
\hline Prognostic signature (high/low) & 265 & 7.355 & $4.378-12.356$ & $<0.001$ & 2.098 & 8.148 & $4.718-14.071$ & $<0.001$ \\
\hline \multicolumn{9}{|l|}{ Validation group $(n=300)$} \\
\hline Age (high/low) & 300 & 1.356 & $0.995-1.849$ & 0.054 & 0.400 & 1.491 & $1.090-2.041$ & 0.012 \\
\hline Gender (male/female) & 300 & 0.997 & $0.719-1.383$ & 0.986 & 0.134 & 1.144 & $0.818-1.599$ & 0.432 \\
\hline AJCC PT (T4,T3/T2, T1) & 300 & 2.200 & $1.613-3.000$ & $<0.001$ & 0.122 & 1.130 & $0.776-1.647$ & 0.523 \\
\hline AJCC PN (N2, N1/N0) & 300 & 3.023 & $1.542-5.927$ & $<0.001$ & 0.378 & 1.459 & $0.712-2.987$ & 0.302 \\
\hline AJCC PM (MX, M1/M0) & 300 & 3.553 & $2.305-5.478$ & $<0.001$ & 0.410 & 1.507 & $0.950-2.392$ & 0.081 \\
\hline AJCC stage (IV, III/II, I) & 300 & 3.410 & $2.366-4.915$ & $<0.001$ & 0.977 & 2.655 & $1.652-4.267$ & 0.000 \\
\hline Prognostic signature (high/low) & 300 & 3.919 & $2.817-5.453$ & $<0.001$ & 1.236 & 3.443 & $2.435-4.866$ & $<0.001$ \\
\hline
\end{tabular}

The median of prognostic signature scores was used as the cut-off value to stratify gastric cancer patients into high risk group and low risk group

AJCC, the American Joint Committee on Cancer; $\mathrm{HR}$, hazard ratio; $\mathrm{Cl}$, confidence interval

demonstrating that the clinical performance of prognosis signature was stable and reliable for prognostic prediction of GC patients. Multivariate Cox regression analyses demonstrated that prognostic signature was an independent risk factor for DFS in both model set and validation set. Thus the thirteen-lncRNA prognosis signature was helpful to identify the patients with high mortality risk and improve the individualized clinical decisionmaking of GC patients.

The current prognostic signature has a good prospect for clinical application. All parameters in the current prognostic signature were generated by gene detection method, indicating that this prognostic model is a noninvasive method and can be used before surgery. Through this prognostic model consisting of thirteen prognostic lncRNAs, doctors and patients can pre-estimate the risk of death in the next 5 years. This prognostic information is valuable for patients to decide whether to receive surgical treatment or not. Ten-group risk stratification chart in the current study demonstrated that patients in lower risk groups had higher survival probability and patients in higher risk groups had lower survival probability.

GAS5-AS1 was an independent prognostic factor for Hepatocellular carcinoma patients and could be considered as a potential prognostic biomarker [23].
The reduced GAS5-AS1 was significantly correlated with larger tumor, higher TNM stage, and lymph node metastasis for non-small cell lung cancer patients [24]. TTTY14 was significantly correlated with overall survival for GC patients and the prognostic value of TTTY14 was independent to other clinical features [25]. LINC00592 was a potential cancer related lncRNA in cervical cancer and might activate the cancer progression through the regulation of transcription or structural integrity [26]. LincRNA C5orf66-AS1 hypomethylation was significantly associated with overall survival in patients with the squamous cell cancer in the head and neck region [27]. The aberrant hypermethylation-mediated downregulation of C5orf66-AS1 might play an important role in gastric cardia adenocarcinoma tumorigenesis and might serve as a potential prognostic biomarker in predicting gastric cardia adenocarcinoma patients' survival [28].

The previous three prognostic signatures calculated the risk scores by using original gene expression values generated on different gene detection platforms and different standardized methods. The different gene detection platforms and standardized methods reduced the repeatability of research results and hindered the clinical application of prognostic signatures in other population. 


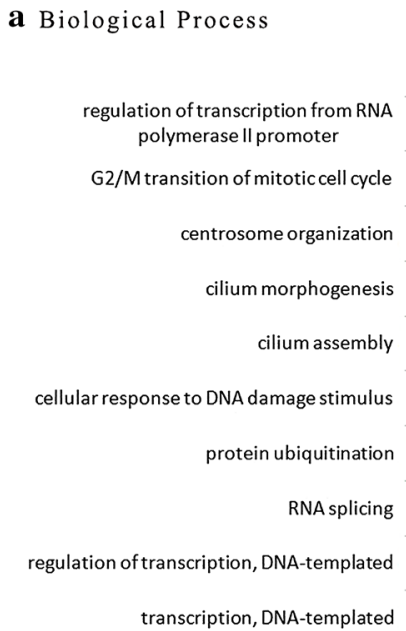

c Cellular Components

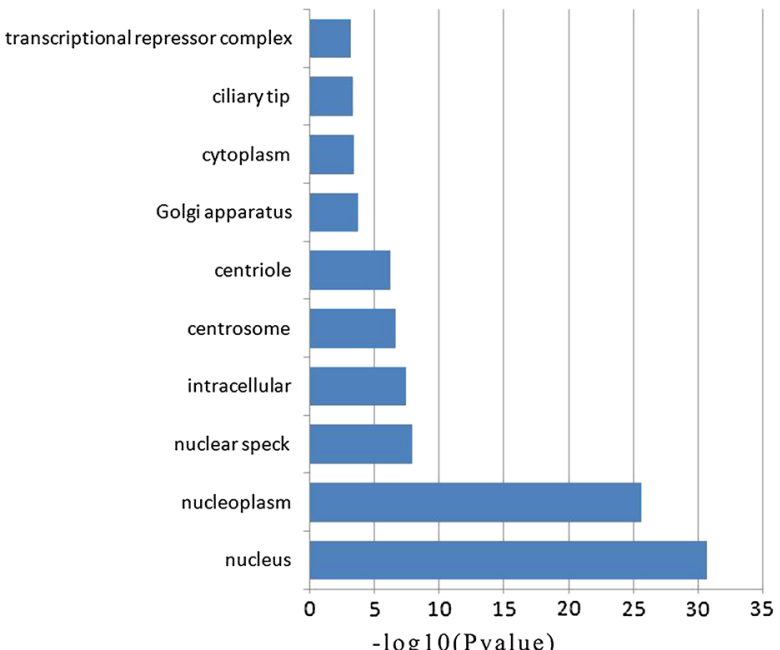

b Molecular Function

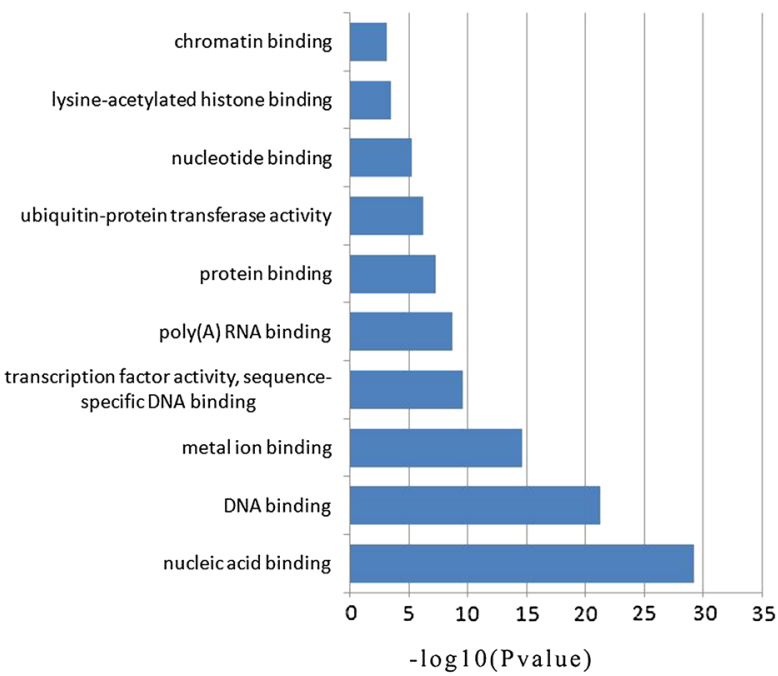

d KEGG Pathway

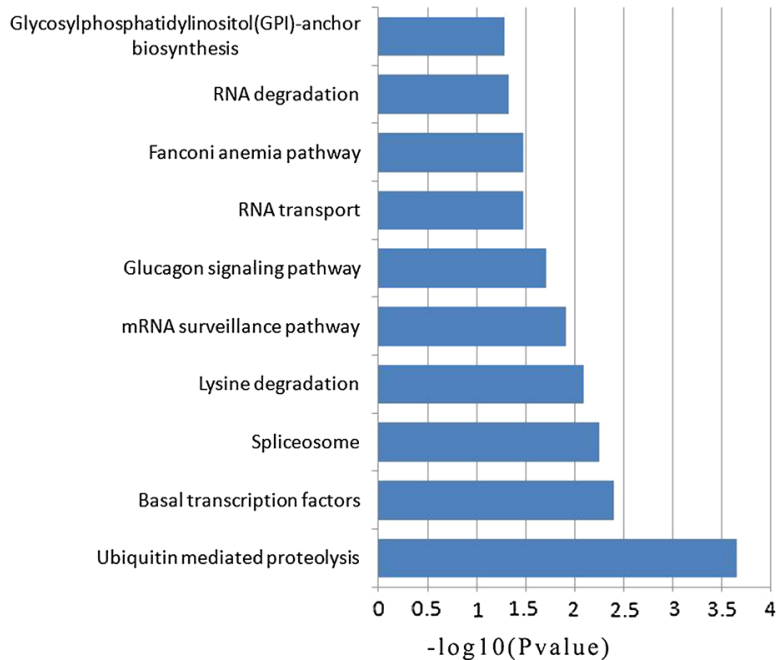

Fig. 9 Functional enrichment analysis of prognostic signature: (a) biological process; (b) molecular function; (c) cellular component; (d) KEGG pathway. KEGG, Kyoto Encyclopedia of Genes and Genomes

Therefore the current study couldn't carry out the previous three prognostic signatures due to the different gene detection platforms and standardized methods. To improve the clinical application of the current prognostic signature in other study population, the thirteen-lncRNA prognostic signature scores in the current study were calculated based on the converted dichotomous values. This dichotomous conversion was helpful to eliminate the obstacles of different detection platforms and standardization methods. Therefore, the thirteen-lncRNA prognostic signature was more suitable for clinical prognostic prediction than the previous three prognostic signatures. 

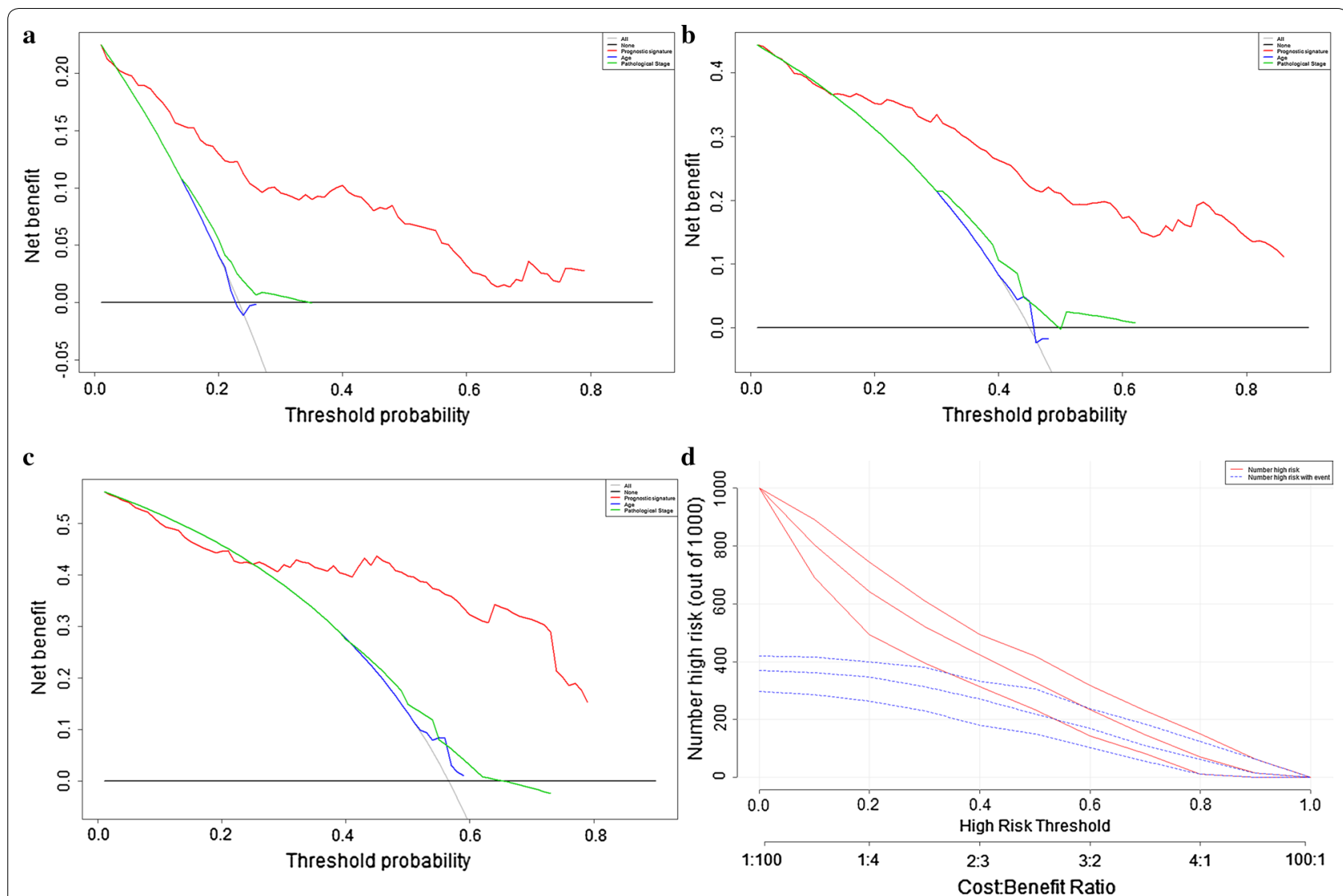

Fig. 10 The decision curve analysis of prognostic signature: decision curve analysis for 1-year disease free survival (a); decision curve analysis for 3-year disease free survival (b); decision curve analysis for 5-year disease free survival (c). The $y$-axis represented the net benefit. The red solid line represented the prognostic signature. The grey solid line represented the net benefit of treating all patients within 1-, 3-, and 5-year. The black solid line represented the net benefit of treating no patients within 1-, 3-, and 5-year

\section{Advantages of the current study}

Firstly, this prognostic nomogram can directly provide the individual mortality percentage forGC patients, which is important for improvement of individualized treatment decision-making. Secondly, the thirteenIncRNA prognostic signature is easy to calculate and understand by users without medical knowledge and professional calculation tool. Thirdly, for patients without pathological diagnosis or unwilling to undergo surgery, the thirteen-lncRNA prognostic signature provides a simple non-invasive preoperative predictive method for prognosis of GC patients, which is of clinical significance for individualized clinical decision-making before surgery.

\section{Limitations of the current study}

As a clinical study by using study datasets downloaded from public databases, the model dataset and validation dataset did not contain detailed study information of drug regimen and other postoperative treatments, which might influence the therapeutic effect and prognosis. Additionally, the results in the current study depended on gene mining approach and lacked evidences from clinical experimental researches. Therefore, it is necessary to carry out large prospective studies to elucidate the relationship between the prognostic lncRNA biomarkers and DFS in GC patients. 

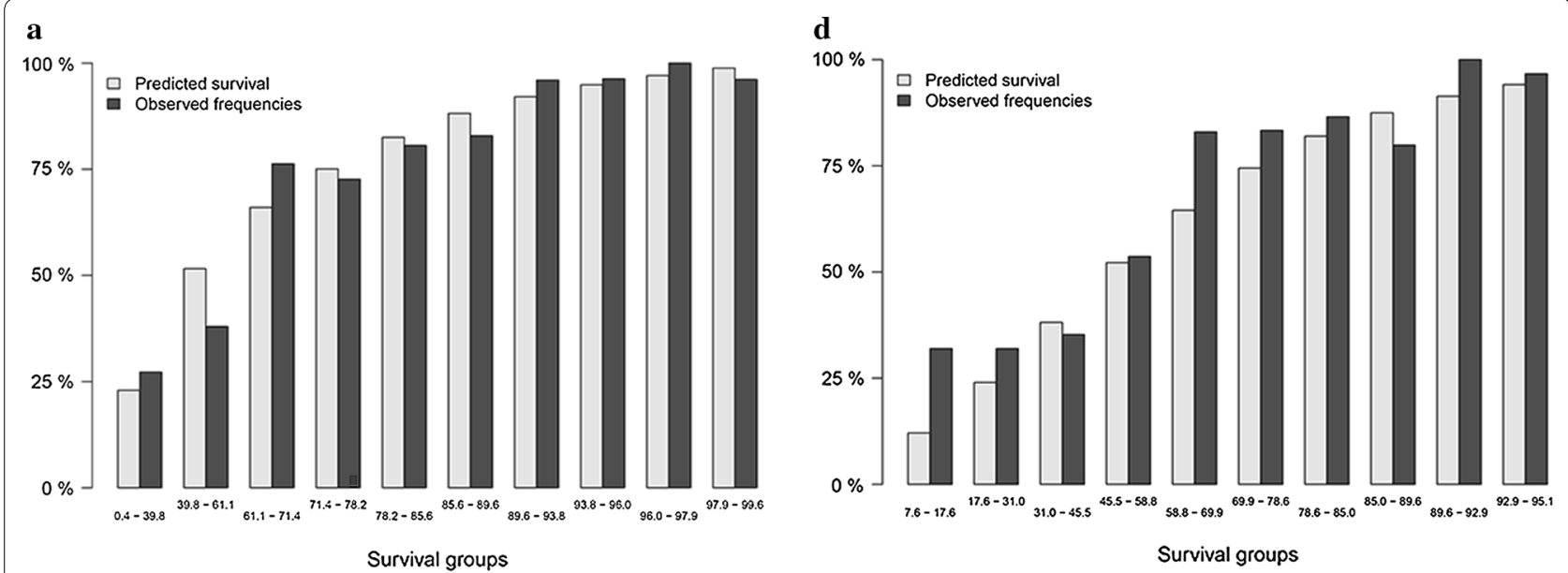

b
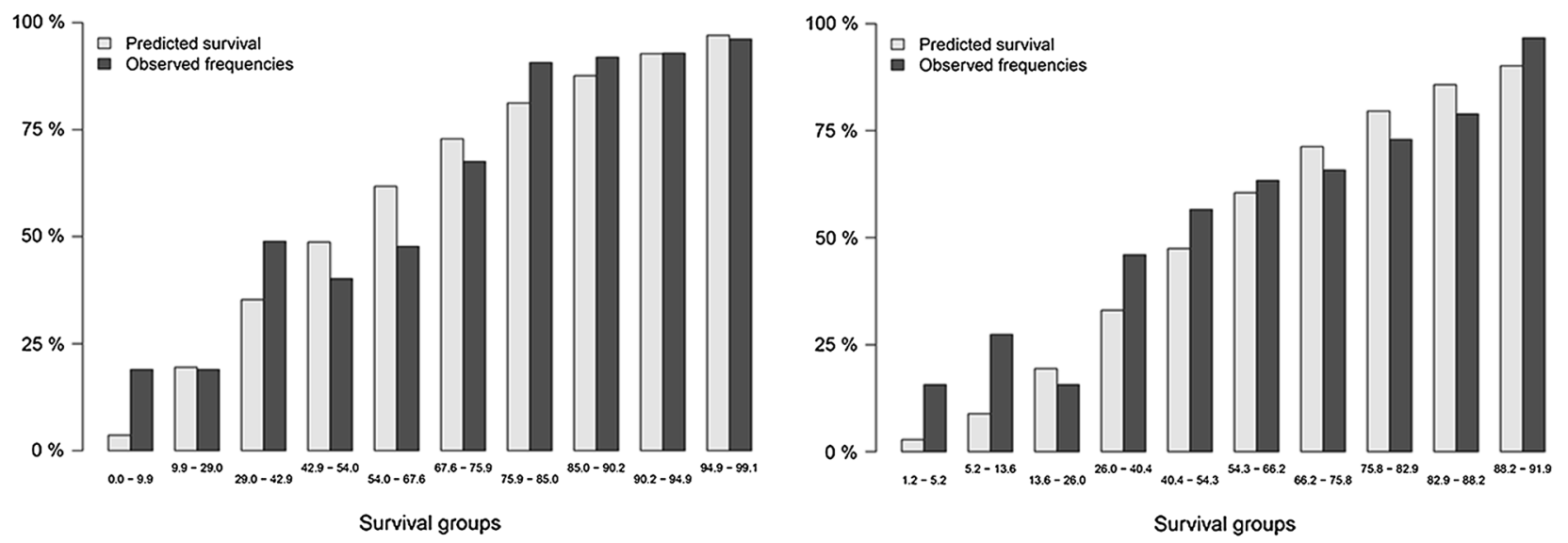

c

f
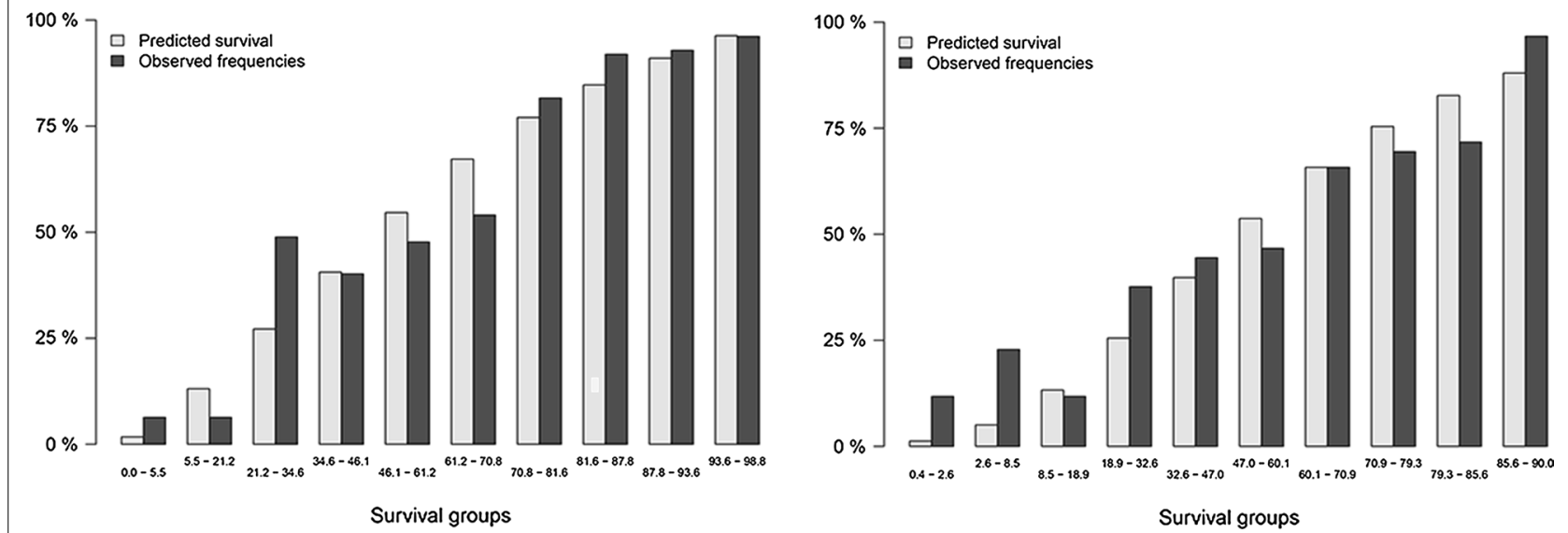

Survival groups

Fig. 11 Ten-group risk stratification chart: (a) for 1-year disease free survival in model group; (b) for 2-year disease free survival in model group; (c) for 3-year disease free survival in model group; (d) for 1-year disease free survival in validation group; (e) for 2-year disease free survival in validation group; (f) for 3-year disease free survival in validation group

\section{Conclusion}

Taken together, a simple noninvasive prognostic signature was established for preoperative prediction of disease free survival in GC patients. This prognostic signature might predict the individual mortality risk of disease free survival without pathological information and facilitate individualized treatment decision-making. 


\section{Additional files}

Additional file 1. Model cohort dataset.

Additional file 2. Validation cohort dataset.

\section{Abbreviations}

GC: gastric cancer; TCGA: The Cancer Genome Atlas; ROC: receiver operating characteristic; DFS: disease-free survival; IncRNA: long non-coding RNA; HR: hazard ratio; Cl: confidence interval; AJCC: the American Joint Committee on Cancer; GEO: the Gene Expression Omnibus; SD: standard deviation; DCA: decision curve analysis.

\section{Acknowledgements}

We appreciated TCGA database and GEO database for providing the original study data. This study was supported by Guangdong Provincial Health Department and Guangdong Provincial Financial Department.

\section{Authors' contributions}

Conceptualization: ZZ, CC, KL and MZ; Data curation: ZZ and QW; Formal analysis: ZZ, MZ, and QW; Funding acquisition: ZZ; Investigation: ZZ, KL, and QW; Methodology: ZZ and CC; Project administration: ZZ, CC and MZ; Resources: ZZ and QW; Software: ZZ and CC; Supervision: ZZ and MZ; Validation: ZZ and QW; Visualization: ZZ, QW and CC; Writing-original draft: ZZ, QW and CC; Writing-review \& editing: ZZ and QW. All authors read and approved the final manuscript.

\section{Funding}

This study was supported by Guangdong Provincial Health Department and Guangdong Provincial Financial Department. The Grant Numbers were: B2018237 and A2016450.

\section{Availability of data and materials}

The datasets analyzed in the current study are provided as the additional documents in the end of the current article.

\section{Ethics approval and consent to participate}

Not applicable.

\section{Consent for publication}

Not applicable.

\section{Competing interests}

The authors declare that they have no competing interests.

\section{Author details}

${ }^{1}$ Department of Internal Medicine, The Affiliated Chencun Hospital of Shunde Hospital, Southern Medical University, Shunde District, Guangdong, China.

${ }^{2}$ Department of General Surgery, The Affiliated Chencun Hospital of Shunde Hospital, Southern Medical University, Shunde District, Guangdong, China.

Received: 19 February 2019 Accepted: 2 May 2019

Published online: 07 May 2019

\section{References}

1. Bray F, Ferlay J, Soerjomataram I, Siegel RL, Torre LA, Jemal A. Global cancer statistics 2018: GLOBOCAN estimates of incidence and mortality worldwide for 36 cancers in 185 countries. CA: A Cancer J Clin. 2018;68:394.

2. Jung KW, Won YJ, Kong HJ, Oh CM, Shin A, Lee JS. Survival of korean adult cancer patients by stage at diagnosis, 2006-2010: national cancer registry study. Cancer Res Treatment. 2013;45(3):162-71.

3. Shah MA, Kelsen DP. Gastric cancer: a primer on the epidemiology and biology of the disease and an overview of the medical management of advanced disease. J Natl Compreh Cancer Netw. 2010;8(4):437-47.
4. Mihmanli M, Ilhan E, Idiz UO, Alemdar A, Demir U. Recent developments and innovations in gastric cancer. World J Gastroenterol. 2016:22(17):4307-20.

5. Marano L, Boccardi V, Braccio B, Esposito G, Grassia M, Petrillo M, Pezzella M, Porfidia R, Reda G, Romano A, et al. Comparison of the 6th and 7th editions of the AJCC/UICC TNM staging system for gastric cancer focusing on the "N" parameter-related survival: the monoinstitutional NodUs Italian study. World J Surg Oncol. 2015;13:215.

6. Zhao L, Jiang L, He L, Wei Q, Bi J, Wang Y, Yu L, He M, Zhao L, Wei M. Identification of a novel cell cycle-related gene signature predicting survival in patients with gastric cancer. J Cell Physiol. 2018;234(5):6350-60.

7. Zhang Y, Li H, Zhang W, Che Y, Bai W, Huang G. LASSObased CoxPH model identifies an 11 IncRNA signature for prognosis prediction in gastric cancer. Mol Med Rep. 2018;18(6):5579-93.

8. Cheng P. A prognostic 3-long noncoding RNA signature for patients with gastric cancer. J Cell Biochem. 2018;119(11):9261-9.

9. McFadden EJ, Hargrove AE. Biochemical methods to investigate IncRNA and the influence of IncRNA: protein complexes on chromatin. Biochemistry. 2016;55(11):1615-30.

10. Zhang W, Song Y. LINC00473 predicts poor prognosis and regulates cell migration and invasion in gastric cancer. Biomed Pharmacother. 2018;107:1-6

11. Wang K, Li J, Xiong YF, Zeng Z, Zhang X, Li HY. A potential prognostic long noncoding RNA signature to predict recurrence among ER-positive breast cancer patients treated with tamoxifen. Sci Rep. 2018;8(1):3179.

12. Zhu M, Wang Q, Luo Z, Liu K, Zhang Z. Development and validation of a prognostic signature for preoperative prediction of overall survival in gastric cancer patients. OncoTargets Ther. 2018;11:8711-22.

13. Zhu X, Tian X, Yu C, Shen C, Yan T, Hong J, Wang Z, Fang JY, Chen H. A long non-coding RNA signature to improve prognosis prediction of gastric cancer. Mol Cancer. 2016;15(1):60

14. Tian X, Zhu X, Yan T, Yu C, Shen C, Hong J, Chen H, Fang JY. Differentially expressed IncRNAs in gastric cancer patients: a potential biomarker for gastric cancer prognosis. J Cancer. 2017:8(13):2575-86.

15. Song P, Jiang B, Liu Z, Ding J, Liu S, Guan W. A three-IncRNA expression signature associated with the prognosis of gastric cancer patients. Cancer Med. 2017:6(6):1154-64.

16. Song W, Miao DL, Chen L. Nomogram for predicting survival in patients with pancreatic cancer. OncoTargets Ther. 2018;11:539-45.

17. Collins GS, Reitsma JB, Altman DG, Moons KG. Transparent reporting of a multivariable prediction model for individual prognosis or diagnosis (TRIPOD): the TRIPOD statement. BMJ (Clinical research ed). 2015;350:g7594.

18. Cristescu R, Lee J, Nebozhyn M, Kim KM, Ting JC, Wong SS, Liu J, Yue YG, Wang J, Yu K, et al. Molecular analysis of gastric cancer identifies subtypes associated with distinct clinical outcomes. Nat Med. 2015;21(5):449-56.

19. Vickers AJ, Cronin AM, Elkin EB, Gonen M. Extensions to decision curve analysis, a novel method for evaluating diagnostic tests, prediction models and molecular markers. BMC Med Inform Decis Mak. 2008:8:53.

20. Vickers AJ, Elkin EB. Decision curve analysis: a novel method for evaluating prediction models. Med Decis Making. 2006;26(6):565-74.

21. Localio AR, Goodman S. Beyond the usual prediction accuracy metrics: reporting results for clinical decision making. Ann Intern Med. 2012;157(4):294-5.

22. Huang DW, Sherman BT, Tan Q, Kir J, Liu D, Bryant D, Guo Y, Stephens R, Baseler MW, Lane HC, et al. DAVID bioinformatics resources: expanded annotation database and novel algorithms to better extract biology from large gene lists. Nucleic Acids Res. 2007;35(Web server issue):W169-75.

23. Wang $Y$, Jing W, Ma W, Liang C, Chai H, Tu J. Down-regulation of long noncoding RNA GAS5-AS1 and its prognostic and diagnostic significance in hep atocellular carcinoma. Cancer Biomark. 2018;22(2):227-36.

24. Wu Y, Lyu H, Liu H, Shi X, Song Y, Liu B. Downregulation of the long noncoding RNA GAS5-AS1 contributes to tumor metastasis in non-small cell lung cancer. Sci Rep. 2016;6:31093.

25. Miao Y, Sui J, Xu SY, Liang GY, Pu YP, Yin LH. Comprehensive analysis of a novel four-IncRNA signature as a prognostic biomarker for human gastric cancer. Oncotarget. 2017;8(43):75007-24. 
26. Yuan $L Y$, Qin $X$, Li L, Zhou J, Zhou M, Li X, Xu Y, Wang XJ, Xing H. The transcriptome profiles and methylation status revealed the potential cancer-related IncRNAs in pa tients with cervical cancer. J Cell Physiol. 2018;234(6):9756-63.

27. Sailer V, Charpentier A, Dietrich J, Vogt TJ, Franzen A, Bootz F, Dietrich D, Schroeck A. Intragenic DNA methylation of PITX1 and the adjacent long non-coding RNA C5orf66-AS1 are prognostic b iomarkers in patients with head and neck squamous cell carcinomas. PLOS ONE. 2018;13(2):e0192742.
28. Guo W, Lv P, Liu S, Xu F, Guo Y, Shen S, Liang J, Kuang G, Dong Z. Aberrant methylation-mediated downregulation of long noncoding RNA C5orf66AS1 promotes the developme nt of gastric cardia adenocarcinoma. Mol Carcinog. 2018;57(7):854-65.

\section{Publisher's Note}

Springer Nature remains neutral with regard to jurisdictional claims in published maps and institutional affiliations
Ready to submit your research? Choose BMC and benefit from:

- fast, convenient online submission

- thorough peer review by experienced researchers in your field

- rapid publication on acceptance

- support for research data, including large and complex data types

- gold Open Access which fosters wider collaboration and increased citations

- maximum visibility for your research: over $100 \mathrm{M}$ website views per year

At BMC, research is always in progress.

Learn more biomedcentral.com/submissions 